\title{
Instrumentation and metrology for single RNA counting in biological complexes or nanoparticles by a single-molecule dual-view system
}

\author{
HUI ZHANG, ${ }^{1,2}$ DAN SHU, ${ }^{1,2}$ FAQING HUANG, ${ }^{3}$ and PEIXUAN GUO ${ }^{1,2}$ \\ ${ }^{1}$ Department of Comparative Pathobiology, Purdue University, West Lafayette, Indiana 47907, USA \\ ${ }^{2}$ Weldon School of Biomedical Engineering, Purdue University, West Lafayette, Indiana 47907, USA \\ ${ }^{3}$ Department of Chemistry and Biochemistry, University of Southern Mississippi, Hattiesburg, Mississippi 39406, USA
}

\begin{abstract}
Limited by the spatial resolution of optical microscopy, direct detection or counting of single components in biological complexes or nanoparticles is challenging, especially for RNA, which is conformationally versatile and structurally flexible. We report here the assembly of a customized single-molecule dual-viewing total internal reflection fluorescence imaging system for direct counting of RNA building blocks. The RNA molecules were labeled with a single fluorophore by in vitro transcription in the presence of a fluorescent AMP. Precise calculation of identical or mixed pRNA building blocks of one, two, three, or six copies within the bacteriophage phi29 DNA packaging motor or other complexes was demonstrated by applying a photobleaching assay and evaluated by binomial distribution. The dual-viewing system for excitation and recording at different wavelengths simultaneously will enable the differentiation of different complexes with different labels or relative motion of each labeled component in motion machines.
\end{abstract}

Keywords: single or dual labeling; bacteriophage phi29 motor pRNA; viral DNA packaging motor; photobleaching

\section{INTRODUCTION}

The resolution in light microscopy, the two closest points that can be distinctly discriminated, is $\sim 200 \mathrm{~nm}$, due to the diffraction limit. The resolving power, $S$, is empirically given as $S=1.22 \lambda /(2 n \sin \theta)$, where $\lambda$ is the wavelength of the light source, $n$ is the refractive index of the immersion oil, and $n \sin \theta$ is the numerical aperture (NA) of the objective. The larger the NA, the higher the quality of the objective. Taking into account the highest NA 1.45 available for oil immersion objectives, the spatial resolution is about half of the wavelength.

Although the law of nature described above cannot be modified, the resolution can be improved by alternative approaches. Scanning tunneling microscopy (STM) (Mikkelsen et al. 2004; Kibsgaard et al. 2006), transmission electron microscopy (TEM) (Kastner et al. 1991; Pante et al. 1997; Fu et al. 2004), scanning electron microscopy

Reprint requests to: Peixuan Guo, Department of Biomedical Engineering, College of Engineering and College of Medicine, University of Cincinnati, Cincinnati, OH 45221, USA; email: peixuan@uc.edu.

Article published online ahead of print. Article and publication date are at http://www.rnajournal.org/cgi/doi/10.1261/rna.587607.
(SEM) (Elias et al. 2005; Yan et al. 2006), atomic force microscopy (AFM) (Gould et al. 1988; Marti et al. 1988; Chen et al. 2000), and optical tweezers (Block et al. 1990; Finer et al. 1994; Svoboda and Block 1994; Johnson et al. 2007) have been used to image nanostructured materials, including nanomotors, with resolution in the nanometer scale. However, direct imaging or counting of biomolecules remains challenging. For example, the application of STM in biomaterials is limited since it relies on the quantum property of the object to generate images. For AFM, although measurement of its height is relatively reliable, the determination of shape and size in $X$ and $Y$ dimensions is limited by the AFM tip size. Direct counting or imaging of individual RNA molecules with TEM or AFM is still challenging due to their versatile conformation and flexible structure.

The single-molecule approach is another alternative in the study of nanomachines and nanoparticles (Rauer et al. 1996; Weiss 1999; Dohoney and Gelles 2001). It has been used to study the activity of enzymes during biochemical reactions (Iwane et al. 1997; Lu et al. 1998), conformational changes of proteins (Sosa et al. 2001) and nucleic acids (Ha et al. 1999, 2002; Zhuang et al. 2000; Joo et al. 2006), and motions of nanomotors (Adachi et al. 2000; Yildiz et al. 2003; Balci et al. 2005) at the single-molecule level. Spatial 
resolution of a few nanometers has been achieved by combining the single-molecule technique with mathematical function fitting (Yildiz et al. 2003; Gordon et al. 2004; $\mathrm{Qu}$ et al. 2004; Balci et al. 2005). In recent years, the singlemolecule technique has been used in studies of RNA folding. Instead of averaging from the ensemble results, the individual events for each single molecule could be studied. The heterogeneity among individual molecules could be revealed (Chu 2003; Xie et al. 2004). A singlemolecule tweezer technique has been applied to measure the persistence length of double-stranded RNA (Abels et al. 2005). With single-molecule fluorescence resonance energy transfer (FRET) using total internal reflection fluorescence (TIRF) microscopy, distinctive dynamic states during the reaction pathway of the hairpin ribozyme can be clearly revealed (Zhuang et al. 2002; Rueda et al. 2004). We report here the assembly of a highly sensitive single-molecule dual-viewing TIRF imaging system (SMDV-TIRF) for the direct counting of RNA molecules labeled with a single fluorophore. We applied a photobleaching assay and binomial distribution analysis to determine the stoichiometry of RNA molecules in nanomachines or biological complexes.

\section{RESULTS AND DISCUSSION}

\section{Assembly of the single-molecule dual-viewing TIRF imaging system (SMDV-TIRF)}

In the imaging system, a laser combiner was used for easy laser beam delivery (Fig. 1). The laser combiner ALC-001, assembled with the assistance of Andor Technology, contains two laser units inside, one of $532 \mathrm{~nm}$ in wavelength and the other of $638 \mathrm{~nm}$ in wavelength (CrystaLaser). The two beams were aligned inside the combiner box to coincide and were directed to an optical fiber out of the box. Both beams can be turned on separately for single-color imaging or simultaneously for dual-color imaging by softwarecontrolled shutters inside the combiner. The use of the laser combiner with the optical fiber saves space, enables quick switching between beams, and allows easy manipulation of the beam. The closed box of the combiner also reduces the possible exposure of eyes to the directed or scattered laser beam during alignment. The use of the fiber to replace the multiple reflecting mirrors greatly facilitated the laser beam alignment. In the photobleaching assay (as described below), a high laser power is not required, and therefore the laser power reduction after fiber delivery is not a concern.

The perfusion chamber for sample incubation and imaging was made of a quartz slide and a glass coverslip (Fig. 1). Two holes were drilled on the quartz slide for infusion of the sample solutions. A $100 \mu \mathrm{m}$ space was generated between the slide and glass coverslip by a spacer. The resultant chamber can hold $\sim 30 \mu \mathrm{L}$ of aqueous solution.

Total internal reflection (TIR) through a quartz prism was utilized to generate the evanescent field at the quartz/
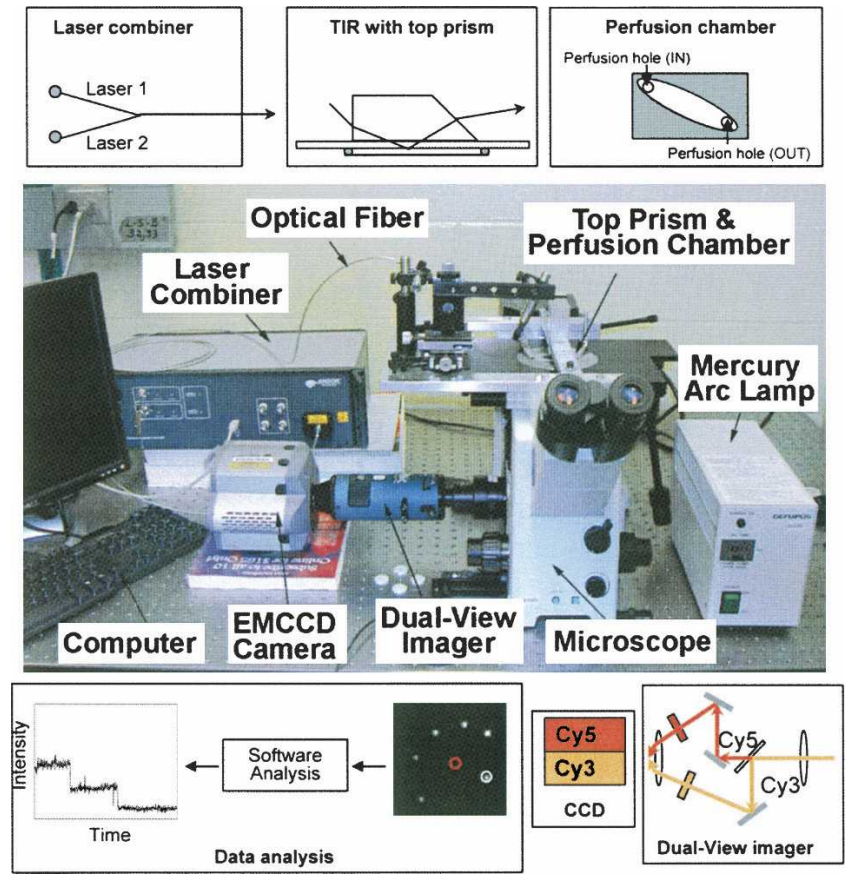

FIGURE 1. Setup of single-molecule dual-viewing TIRF imaging system (SMDV-TIRF). After the fluorescence image was recorded on the CCD chip of the camera, the data were analyzed by the computer software and converted to an intensity versus time plot to show photobleaching steps. The typical photobleaching trace was obtained by subtracting the background time trajectory of a dark region (red circle) from that of a fluorescent spot (white circle).

aqueous solution interface. When imaging fluorescently labeled samples close to the quartz surface, the fast exponential decay of the laser illumination from TIR greatly reduces the fluorescence background from the bulk solution. TIR fluorescence microscopy (TIRFM) has demonstrated its ability in detecting single fluorophores (Funatsu et al. 1995; Vale et al. 1996; Tokunaga et al. 1997; Xu and Yeung 1997; Ambrose et al. 1999; Zhuang et al. 2000; He et al. 2005). Instead of the objective-type TIRFM (Tokunaga et al. 1997; Ambrose et al. 1999), prismtype TIRFM (Funatsu et al. 1995; Ambrose et al. 1999; Zhuang et al. 2000) was used in the system reported here. The beam was reflected away from the objective after total internal reflection by the prism. This avoids the possible background from the laser beam leaking to the objective during fluorescence collection. The geometry of the top prism also reduces the unstable signals from the loosely attached materials on the quartz surface.

The incident angle of the laser beam at the left side of the prism was set to be $\sim 33^{\circ}$. After refraction through the prism as shown in Figure 1, the incident angle at the quartz/buffer interface was $68^{\circ}$. The penetration depth of the evanescent field was $\sim 160 \mathrm{~nm}$ at this incident angle for the $532 \mathrm{~nm}$ laser, according to $d=\lambda /\left[4 \pi\left(n_{1}^{2} \sin \theta^{2}-n_{2}^{2}\right)^{1 / 2}\right]$, in which $n_{1}$ and $n_{2}$ are the refractive indexes $\left(n_{1}=1.46\right.$ for quartz; $n_{2}=1.33$ for water) and $\theta$ is the incident angle $\left(68^{\circ}\right)$. 
Immersion oil was used to mediate the quartz prism with the quartz slide of the perfusion chamber. Only the fluorophores that were within $160 \mathrm{~nm}$ of the quartz surface could be excited.

Fluorescence signals were collected through the objective and imaged by an electron-multiplied CCD camera. The operating temperature of the camera was set to $-70^{\circ} \mathrm{C}$ when imaging. This extremely low operating temperature enables the reduction of the thermal-dependent dark noise. The use of an electron multiplier in the camera also enhances the sensitivity and speed at low-light imaging.

A dual-view imager was inserted between the microscope and the camera for simultaneous collection of dual-color fluorescence signals. The imager separates the field of view of the CCD chip in the camera into two different channels for signals of different wavelengths. With the proper dual-view filter set (Optical Insights, OI-06-EM; dichroic mirror 630, Cy3 barrier filter 585/30, Cy5 barrier filter 680/35), the imager separates the signals of Cy3 and Cy5 from each other. The Cy5 signal is transmitted through the dichroic mirror inside the imager and reflected by the side mirror to the Cy5 channel, while the Cy3 signal is reflected by the dichroic and directed to the Cy3 channel (Fig. 1; Shu et al. 2007).

\section{Photobleaching assay for quantification of fluorescent nanoparticles}

The $\phi 29$ (phi29) motor has been extensively studied as a model system for viral dsDNA packaging. The packagingRNA (pRNA) of the motor interacts with other motor proteins and facilitates the DNA packaging. The pRNA has also been demonstrated to have applications in nanotechnology. The length of the pRNA and the size of the entire motor are shorter or smaller than $40 \mathrm{~nm}$, which is beyond the optical resolution to quantify the copy number of pRNA within a single complex. We developed the fluorescence photobleaching assay for quantification of fluorescent pRNA within these nanoparticles (Shu et al. 2007). The pRNA molecule was labeled with a single Cy3 or Cy5 at its $5^{\prime}$ end ( $\mathrm{Li}$ et al. 2005). The in vitro assembly test showed that such labeled pRNA was active in DNA packaging and $\phi 29$ virion assembly.

In the photobleaching assay of the fluorescent nanoparticles, the sample that was immobilized to the surface of the perfusion chamber appeared as discrete bright spots (Fig. 1). Continuous excitation with the laser beam causes the fluorescence intensity of the spots to decrease over time. Andor IQ software provides analysis of average intensity within a region versus time. A circled area around the fluorescent spot (white circle) was chosen as illustrated in data analysis in Figure 1. The mean fluorescence intensity of the circled area was calculated by the software and plotted with time after subtracting background mean intensity (red circle). Quantized photobleaching is charac- teristic of single fluorophores, and the time required for each fluorophore to be photobleached follows a Poisson distribution. Hence, the number of photobleaching steps represents the number of fluorescent molecules within one spot, which cannot be spatially resolved by a conventional optical microscope. Even though the purpose is to observe a photobleaching event, photobleaching that is too fast may result in poor temporal resolution and difficulty in the determination of the steps. Hence, an oxygen depletion solution (Ha et al. 2002) was used to impede the photodynamic photobleaching process in the presence of oxygen.

The key in the determination of the steps is to select spots that are completely bleached and disappear from the field. In addition, the intensity should reach the lowest level after an extended time of laser beam illumination. Only those experimental data spots that fulfill the above criteria were used for statistical analysis to ensure that the steps reflect the correct number of fluorophores.

The baseline of the photobleaching curve was derived from background areas selected with the same size as the spot. Due to the Gaussian distribution of laser beam intensity, sometimes small variations in background intensity can occur, depending on the selected area. It was found that reducing the size of the circle around the spot and taking the average of multiple circles close to the fluorescent spot as background for analysis produced clearer traces of photobleaching steps (Fig. 2). When the area of

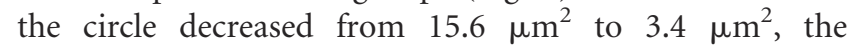
beginning fluorescent intensity of the same spot increased from 50 arbitrary units (A.U.) (Fig. 2A) to 150 A.U. (Fig. $2 \mathrm{C}$ ), relative to the background within the same sized circle accordingly. The signal-to-noise ratio was increased and the photobleaching steps appeared clearer, but the determination of the number of steps was not affected.

\section{Quantification of fluorescent pRNA within His-gp16/pRNA nanoparticles}

To evaluate the feasibility of this system for counting nanoparticles containing one or two fluorophores attached, the photobleaching assay was first applied to study the

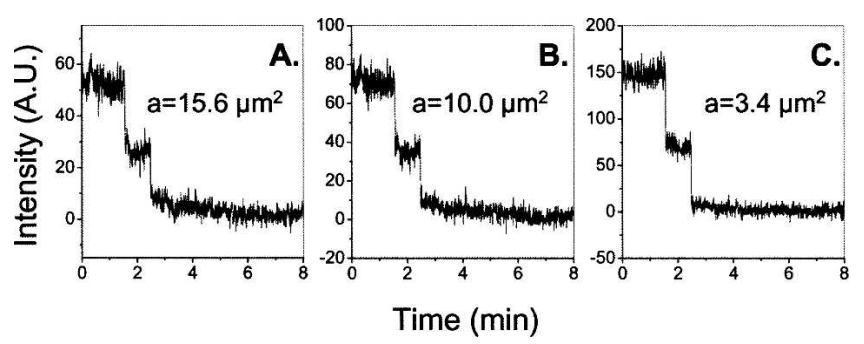

FIGURE 2. Comparison of the time trajectory of relative average intensity for the same fluorescent spot when using circles of different sizes in software analysis. (A) The area of the circle $a=15.6 \mu \mathrm{m}^{2}$; (B) $a=10 \mu \mathrm{m}^{2}$; (C) $a=3.4 \mu \mathrm{m}^{2}$. 
nanoparticles of His-gp16/pRNA complexes. Gp16 is one of the essential components of the $\$ 29$ motor. His-gp16, which is the gp16 with a six-histidine tag (Huang and Guo 2003b), has the same biological activity in infectious virion assembly. It binds to pRNA at the pRNA's double-helix domain (Lee and Guo 2006). Two different complexes were assembled with monomeric Cy3-pRNA, or dimeric Cy3pRNA (Fig. 3I,II, panels A). The His-gp16/pRNA complexes were immobilized to the surface of the quartz chamber through anti-His-gp16 IgG. The number of photobleaching steps for each fluorescent spot was obtained and summarized as the percentage in the population (Fig. 3I,II, panels B). Differences were found in the distribution of photobleaching steps for these two different complexes. The majority of the spots showed one step in photobleaching for the complexes formed from monomeric Cy3-pRNA, while in the complex of dimeric Cy3-pRNA, the counts in one- and two-step photobleaching were almost equal. The histograms in photobleaching steps for the two samples are distinguishable from each other. The distribution was inclined to a higher number of steps for the complexes composed of dimers, compared to that of monomers.

The reliability of the finding was further tested by statistical analysis using a binomial distribution formula. The number of photobleaching steps for each spot represents the number of fluorescent pRNAs within a complex (e.g., the motor). However, it does not directly reflect the number of total pRNAs bound because of the incomplete labeling of pRNA due to the technical limit in preparation of fluorophore-labeled pRNA. The possibility of observing different numbers of steps in photobleaching, which also represents that of observable labeled pRNA within each complex, can be modeled with a binomial distribution. If the labeling efficiency is expressed as a fraction $p$, and the portion of unlabeled pRNA is $q$, then $q=(1-p)$, and $(p+$ $q)=1(100 \%)$. The distribution of the copy number of labeled pRNAs follows the expansion of the binomial

$$
\begin{aligned}
(p+q)^{Z}= & \left(\begin{array}{c}
Z \\
0
\end{array}\right) p^{Z}+\left(\begin{array}{c}
Z \\
1
\end{array}\right) p^{Z-1} q+\ldots\left(\begin{array}{c}
Z \\
i
\end{array}\right) p^{Z-i} q^{i}+\ldots \\
& +\left(\begin{array}{c}
Z \\
Z-1
\end{array}\right) p q^{Z-1}+\left(\begin{array}{c}
Z \\
Z
\end{array}\right) q^{Z}
\end{aligned}
$$

where $Z$ is the total number of pRNA molecules that each nanoparticle, nanomotor, or nanodevice holds and $i$ is the number of pRNA molecules that do not contain fluorophores. The binomial coefficient $\left(\begin{array}{l}Z \\ i\end{array}\right)$ represents the number of possible combinations for $Z$ pRNA molecules to have $(Z-i)$ labeled pRNA and $i$ unlabeled pRNA, which is equal to

$$
Z ! /[i !(Z-i) !]
$$

The determination of probability can be followed by the formula of Yang Hui triangle (Pascal's triangle) (Fig. 4).
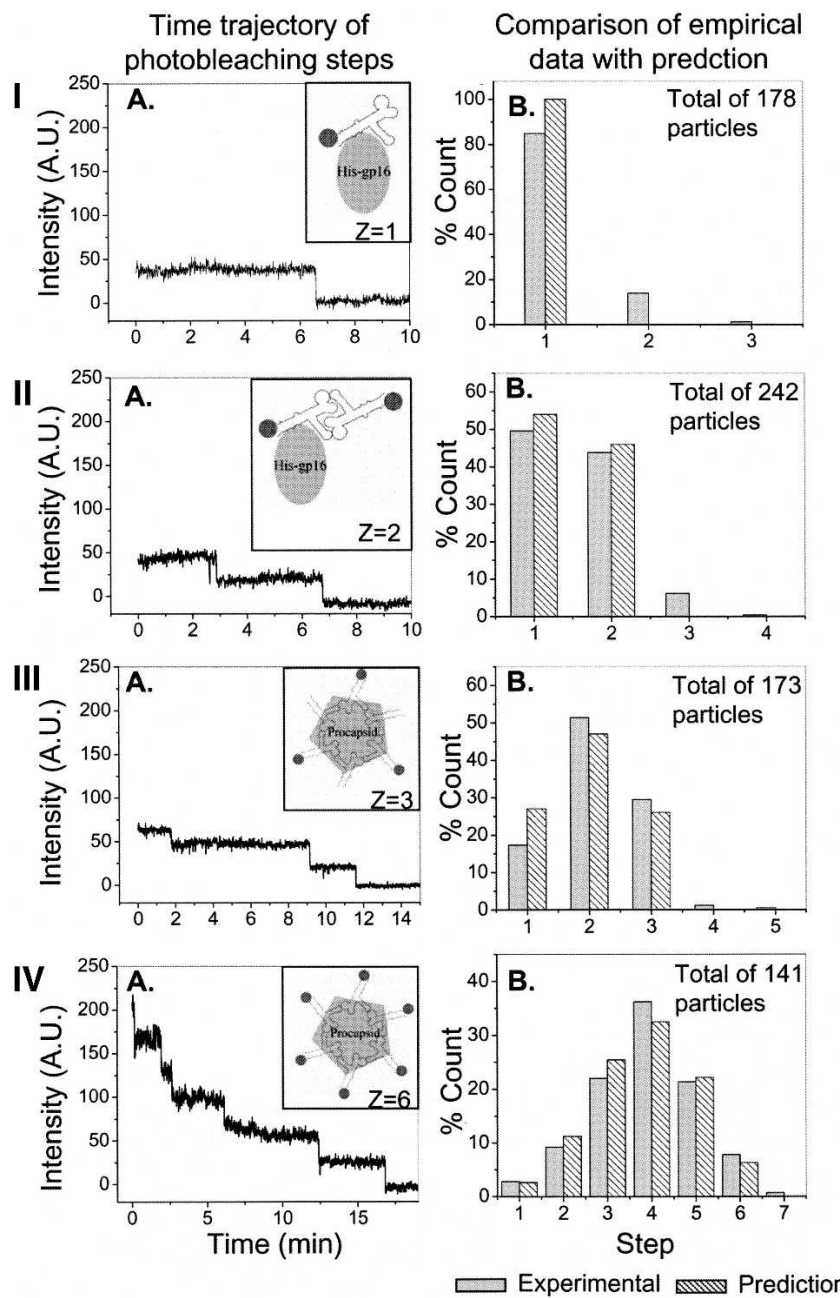

FIGURE 3. Analysis of fluorescent particles composed of different protein/pRNA complexes. The insets depict the assembly of the different protein/pRNA complexes. The dark gray dot represents Cy3 label to pRNA at $5^{\prime}$ end. $Z$ indicates the copy number of labeled pRNA within each complex. The fluorescence intensity is shown in arbitrary units (A.U.). The theoretical predictions were based on $63 \%$ Cy3 labeling efficiency. (A) His-gp16/ monomeric Cy3-pRNA complex $(Z=1)$. (B) His-gp16/ dimeric Cy3-pRNA complex $(Z=2)$. (C) Procapsid/pRNA complex made from single Cy3-labeled pRNA dimer; each resulting nanoparticle contains three labeled pRNAs $(Z=3)$. (D) Procapsid/pRNA complex made from homogeneous Cy3-pRNA; each resulting nanoparticle contains six labeled pRNAs $(Z=6)$.

For example, if each nanoparticle holds two pRNA molecules in total, $Z=2$. The probability of the nanoparticle containing only one labeled pRNA and one unlabeled pRNA is therefore equal to $\left(\begin{array}{l}2 \\ 1\end{array}\right) p^{2-1} q$, which is $2 p q$. The probability of the nanoparticle having both Cy3-labeled pRNAs can be calculated from $\left(\begin{array}{l}2 \\ 0\end{array}\right) p^{2}$, which is $p^{2}$; and the probability of the nanoparticle having both unlabeled pRNAs would therefore be $q^{2}$.

When using $(p+q)^{Z}$ to predict the distribution of nanoparticles constructed with monomeric Cy3-pRNA (Fig. $3 \mathrm{~A}$ ), $Z$ is equal to 1 . Since fluorescence microscopy 


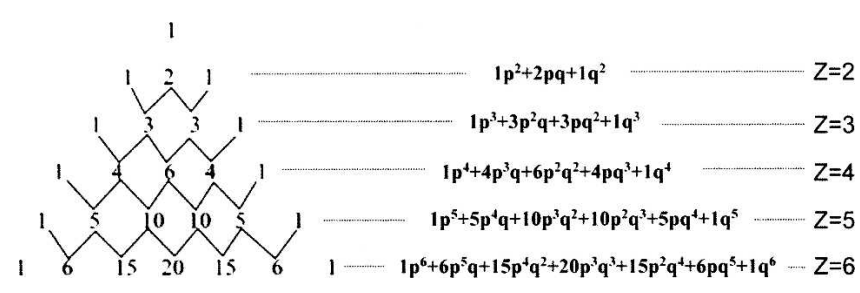

FIGURE 4. Yang Hui triangle (Pascal's triangle) for binomial coefficient.

can only detect the labeled pRNA, theoretically $100 \%$ of the observable particles should have only one Cy3 attached. Figure 3I, panel B, shows the comparison between the prediction and the experimental data. There are $\sim 14 \%$ of the particles that showed two-step photobleaching, which represents two $\mathrm{Cy} 3$ molecules attached, suggesting some aggregation of these nanoparticles.

When using $(p+q)^{Z}$ to predict the distribution of nanoparticles constructed with dimeric Cy3-pRNA (Fig. $2 \mathrm{~B}), Z$ is equal to 2 . As discussed earlier, the probability of the particles that contain two Cy3 molecules is equal to $p^{2}$; the probability of the particles that contain only one $\mathrm{Cy} 3$ is equal to $2 p q$; and the probability of the particles that have no Cy3 label is equal to $q^{2}$. As determined by UV/Vis absorbance (see Materials and Methods), $p$, the labeling efficiency of $\mathrm{Cy} 3$ for the pRNA, is $63 \% \pm 5 \%$. The $5 \%$ is the standard deviation from the measurement. Thus $p$ equals 0.63 and $q$ is 0.37 . Therefore, the theoretical distribution for the possibility of the nanoparticles having two Cy 3 molecules attached predicted from the Equation 1 is $p^{2} /\left(p^{2}+2 p q\right)$, which is 0.54 . The possibility of the particles having one Cy3 molecule attached is $2 p q /\left(p^{2}+2 p q\right)$, which equals 0.46 . Figure $3 \mathrm{II}$, panel $\mathrm{B}$, showed that the experimental data fit well with the prediction.

\section{Quantification of fluorescent pRNA within \$29 nanomotors}

To evaluate the reliability of the photobleaching assay for the determination of stoichiometry of nanoparticles or nanomachines that contain three or six copies of the component, fluorescent pRNAs were assembled onto $\phi 29$ procapsids and formed nanomotors with three or six fluorescent pRNAs, respectively, in each particle (Fig. 3III,IV, panels A). It has been shown that the $\$ 29$ DNA packaging motor binds six copies of pRNA (Chen and Guo 1997; Guo et al. 1998; Zhang et al. 1998; Ibarra et al. 2000; Fang et al. 2005; Shu et al. 2007). The motor can also be assembled from six monomeric pRNAs, three dimeric pRNAs, or two trimeric pRNAs (Guo et al. 1998; Zhang et al. 1998; Chen et al. 2000; Hoeprich and Guo 2002; Fang et al. 2005). When assembled from a homogeneous fluorescent monomer, the resultant nanomotor contains six labeled pRNAs. By labeling only one pRNA within each dimeric pRNA, each nanomotor theoretically contains three labeled pRNA molecules and three unlabeled pRNA molecules. The histograms obtained experimentally from the photobleaching assay for the two different motor complexes are shown in Figure 3, III (panel B) and IV (panel B), with the difference in the range of the distribution and in the step number that has the highest fraction.

Similarly, $(p+q)^{Z}$ was used to predict the distribution in photobleaching steps, where for nanomotors containing three Cy3-pRNAs and three unlabeled pRNAs, $Z$ equals 3. The distribution follows the expansion of $(p+q)^{3}$, which is $\left(p^{3}+3 p^{2} q+3 p q^{2}+q^{3}\right)$. The probability of the particles that contain three $\mathrm{Cy} 3$ molecules in the population of observable particles is $p^{3} /\left(p^{3}+3 p^{2} q+3 p q^{2}\right)=0.27$; the probability of the particles that contain only two Cy3 molecules is $3 p^{2} q /\left(p^{3}+3 p^{2} q+3 p q^{2}\right)=0.47$; and the probability of the particles that contain only one $\mathrm{Cy} 3$ molecule is $3 p q^{2} /\left(p^{3}+3 p^{2} q+3 p q^{2}\right)=0.26$. The predicted pattern of the distribution is close to that obtained experimentally (Fig. 3III, panel B).

The distribution in photobleaching steps for nanomotors constructed from six monomeric Cy3-pRNAs can also be predicted in the same way, with $p$ equal to $0.63, q$ equal to 0.37 , and $Z$ equal to 6 . The possibilities calculated from Equation 1 for nanoparticles having one, two, three, four, five, or six $\mathrm{Cy} 3$ molecules attached were therefore 0.03 , $0.11,0.25,0.33,0.22$, and 0.06 , respectively. The prediction was compared with the experimental data in Figure 3IV, panel B.

Different theoretical histograms are shown in Figure 5 for different labeling efficiencies. The outcome of the histogram greatly depends on the labeling efficiency. There was $\sim 5 \%$ error in the measurement of labeling efficiency by UV/Vis absorbance. The difference of $5 \%$ in labeling efficiency could lead to different distributions in the photobleaching step. However, as shown in Figure 5D, even with a $5 \%$ error in labeling efficiency, a conclusion of either $Z=5$ or $Z=6$ can be easily drawn from the experimental data since the distribution patterns of the steps are distinctively different from each other. When $Z=$ 5 with $70 \%$ labeling efficiency, there is no count at six steps in the histogram. However, when $Z=6$ with $70 \%$ labeling efficiency, there are significant counts at six steps (Figs. 5D, $6)$. Therefore, the conclusion in the stoichiometry of the pentamer or hexamer can be drawn from this method even with a $5 \%$ error in labeling efficiency.

\section{Dual-color imaging of multiple-labeled pRNA within ф29 nanomotors}

Installed with the dual-view imager, the SMDV-TIRF system also includes the dual-color imaging function that can produce an image with two colors or count two types of components, each of which was labeled with one distinctive fluorophore. As noted above, the pRNA ring 
A $\quad \mathrm{Z}=2$

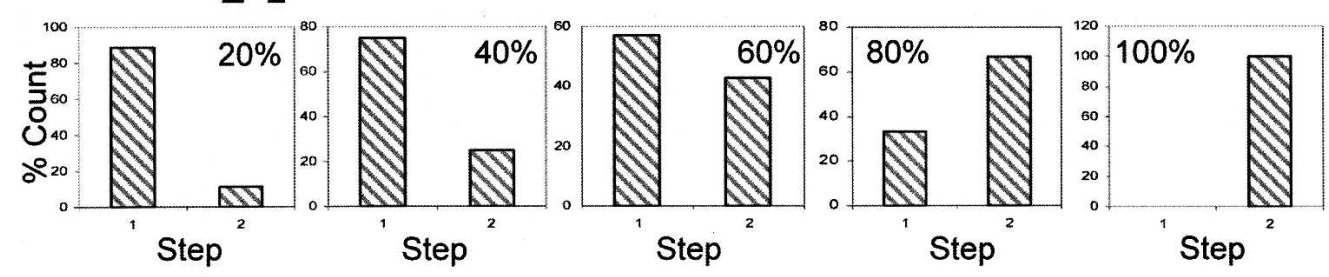

B $\quad Z=3$

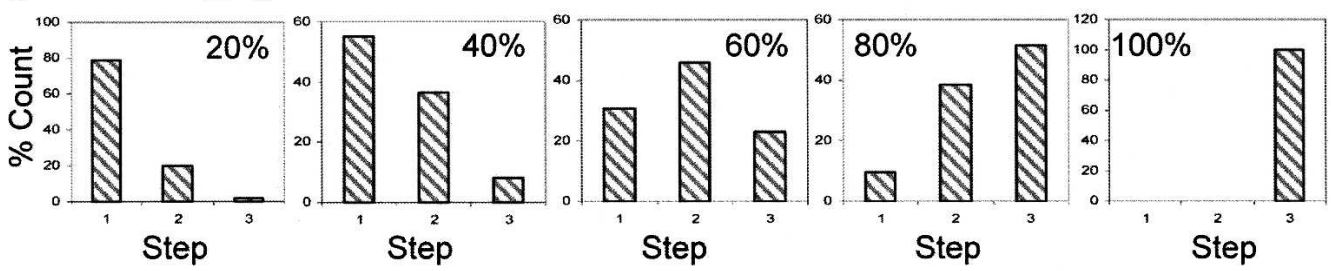

C $\quad \mathrm{Z}=6$
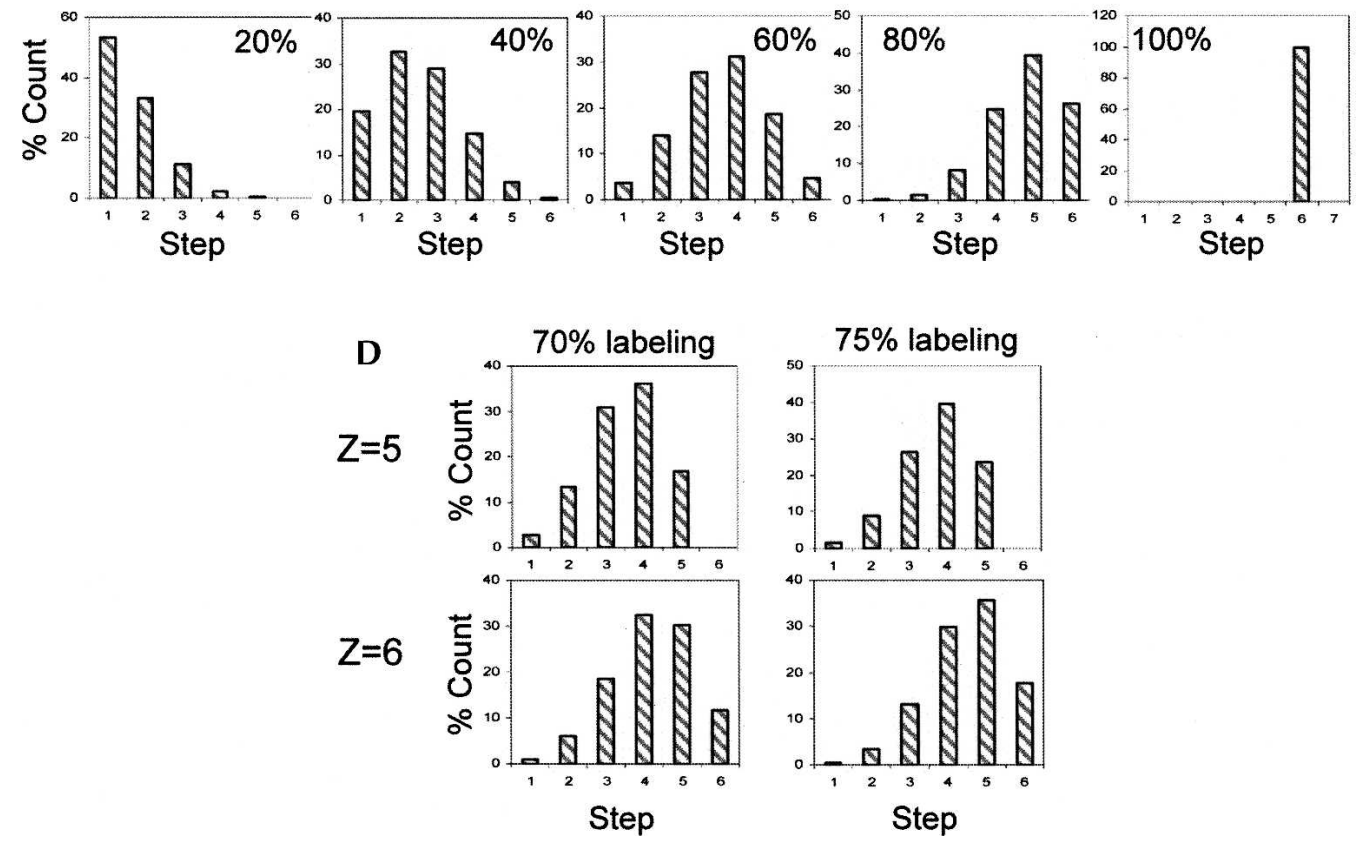

FIGURE 5. Theoretical histograms of photobleaching step distributions at different labeling efficiencies of 20\%, 40\%, 60\%, 80\%, and 100\%. (A) Models for $Z=2$. (B) Models for $Z=3$. (C) Models for $Z=6$. (D) Comparison of theoretical models for $Z=5$ and $Z=6$ at $70 \%$ and $75 \%$ labeling efficiencies, respectively.

of the $\phi 29$ DNA packaging motor is composed of six pRNA molecules (Chen and Guo 1997; Guo et al. 1998; Zhang et al. 1998; Ibarra et al. 2000; Fang et al. 2005; Shu et al. 2007), which can be constructed using six monomers, three dimers, or two trimers as building blocks via the hand-inhand interaction of the left and right interlocking loops (Guo et al. 1998; Zhang et al. 1998; Chen et al. 2000; Hoeprich and Guo 2002; Fang et al. 2005). By labeling each pRNA molecule in the building block with a different fluorophore, a motor with a multilabeled pRNA hexamer can be obtained (Shu et al. 2007). Figure 7A shows the typical dual-color fluorescent image of the nanomotors. Part of the six pRNA molecules on the hexameric ring were labeled with $\mathrm{Cy} 3$ and the other pRNA molecules on the ring were labeled with Cy5. Both fluorophores were excited simultaneously by dual laser $(532 \mathrm{~nm}$ for Cy3 and $638 \mathrm{~nm}$ for Cy5) illumination. The signals were collected and then split by the dual-view imager to the Cy 3 and Cy5 channels, respectively. Pseudocolors for each fluorophore were used, with green representing $\mathrm{Cy} 3$ and red representing $\mathrm{Cy} 5$ (Fig. 7A). The resultant dual-view image was subjected to the field-splitting process by the Andor IQ to superimpose the images in the two channels. The yellow spots result from the overlay of red and green. The red spots represent the procapsid/pRNA complexes containing only Cy5 fluorophores, resulting from the combination of Cy5-pRNAs 

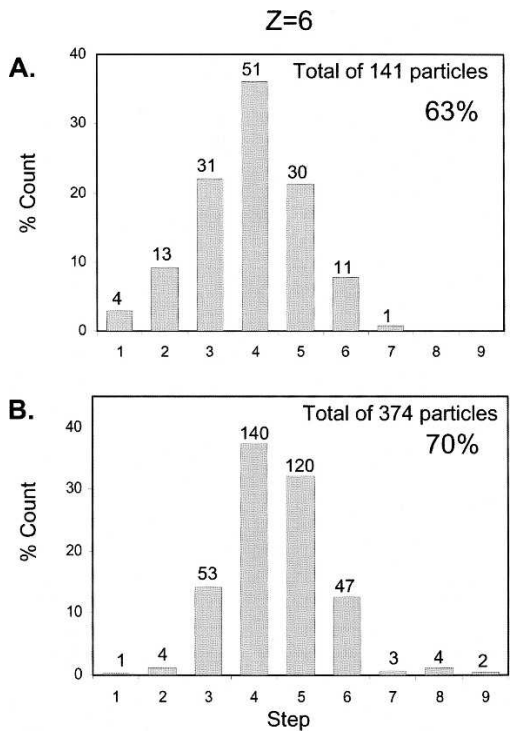

FIGURE 6. Comparison of the experimental data of pRNA with $(A)$ $63 \%$ labeling efficiency and (B) 70\% labeling efficiency for particles containing six Cy3-pRNA molecules. The number above each column indicates the actual count.

with unlabeled pRNA counterparts. Similarly, the green spots represent the procapsid/pRNA complexes containing only Cy3 fluorophores, resulting from the combination of Cy3-pRNA with unlabeled pRNA counterparts. When the hexameric pRNA ring was constructed using Cy3-pRNA only, the signals only appeared in the $\mathrm{Cy} 3$ channel after dual laser excitation (Fig. 7B). Overlaid images from both Cy3 and Cy5 channels of pRNA rings with only Cy3-pRNA resulted in only green spots. When the hexameric pRNA ring was constructed using Cy5-pRNA only, the signals only appeared in the $\mathrm{Cy} 5$ channel after dual laser excitation (Fig. 7C), and overlaid images from both $\mathrm{Cy} 3$ and $\mathrm{Cy} 5$ channels resulted in only red spots.

Not all the spots appeared to have the yellow overlays in Figure $7 \mathrm{~A}$ due to the incomplete labeling of pRNA. The probability of green spots in the overlaid image can be estimated by the formula $\left[\left(1-q^{Z}\right)-\left(1-q^{Z}\right)\left(1-n^{Z}\right)\right]$ or $\left(1-q^{Z}\right) n^{Z}$, where $q=1-p$, representing the fraction of unlabeled RNA, and $p$ is the labeling efficiency of Cy3-RNA; and $n=1-m$, while $m$ is the labeling efficiency of Cy5-RNA. Similarly, the probability of red spots can be estimated by $\left[\left(1-n^{Z}\right)-\left(1-q^{Z}\right)\left(1-n^{Z}\right)\right]$ or $\left(1-n^{Z}\right) q^{Z}$, and the probability of yellow spots is $\left(1-q^{Z}\right)\left(1-n^{Z}\right)$. When both labeling efficiencies $p$ and $m$ are $100 \%$, the probability of green spots calculated from the formula is $\left(1-q^{Z}\right) n^{Z}=\left(1-0^{Z}\right) 0^{Z}=0$. There would be no green spots, and every spot is yellow from the overlay. If the Cy3 labeling efficiency $p$ is $100 \%$ and for Cy5 labeling efficiency $m$ is $0 \%$, the probability of green spots is $\left(1-q^{Z}\right) n^{Z}=\left(1-0^{Z}\right) 1^{Z}=100 \%$. That is, there would be no yellow spots, and all the spots would be green.
Similar to the significance level testing in statistics, a large number, hundreds to thousands, of spot countings is needed to obtain a reliable parameter. Counting of the red or green spots from one field of view could not be sufficient to reach a conclusion. Since each spot is from a single molecule or a single particle, the collection of hundreds or thousands of spots for data analysis is not difficult in this methodology.

Fluorescence signals were collected in the same manner as that for single-labeled motors, with both signals from

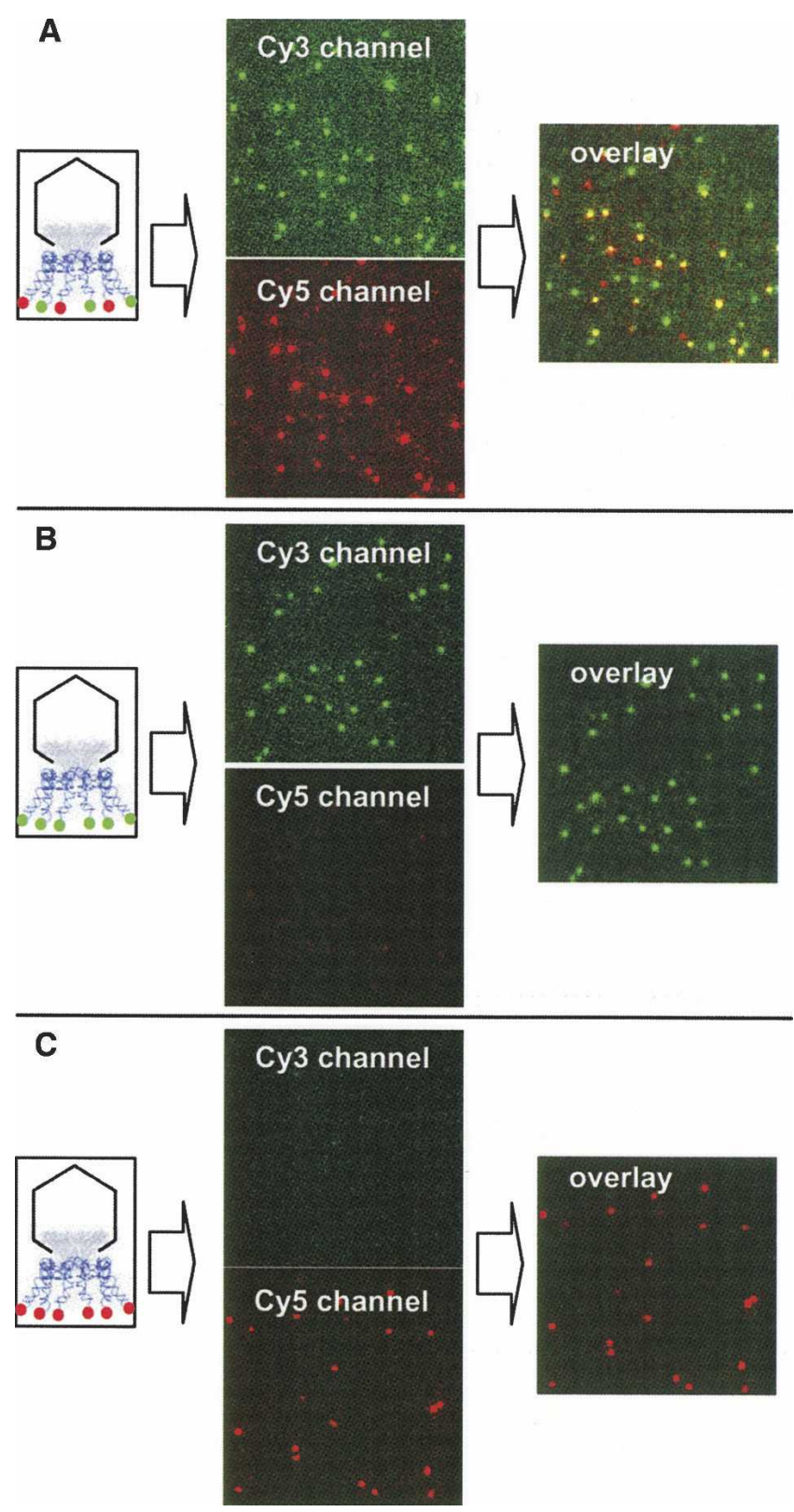

FIGURE 7. Dual-color fluorescence images of $(A)$ the procapsid/ pRNA complexes containing dual-labeled pRNA, $(B)$ the procapsid/ pRNA complexes containing only Cy3-labeled pRNA, and $(C)$ the procapsid/pRNA complexes containing only Cy5-labeled pRNA, as depicted. The fluorescence images are shown in pseudocolor. The images on the right are the overlap from the two channels. 
Cy3 and Cy5 recorded simultaneously. After the field-split process, the mean intensities of the yellow spots were plotted versus time after subtraction of the background. The typical photobleaching curves are shown in Figure 8, with Cy3 in green and Cy5 in red, respectively. The number of photobleaching steps for both Cy3 and Cy5 was distinguished. Steps in the green or red curve indicate the number of Cy3-pRNAs or Cy5-pRNAs, respectively. For example, the plot showing five red steps plus one green step came from the motor containing five Cy5-pRNAs and one Cy3-pRNA (Fig. 8A). The plot showing two red steps plus four green steps came from the motor containing two Cy5pRNAs and four Cy3-pRNAs (Fig. 8B). The plots showing three red steps plus three green steps came from the motor containing three Cy5-pRNAs and three Cy3-pRNAs (Fig. $8 \mathrm{C})$. As Cy3 is more photostable than Cy5 under continuous laser illumination, Cy5 was photobleached much
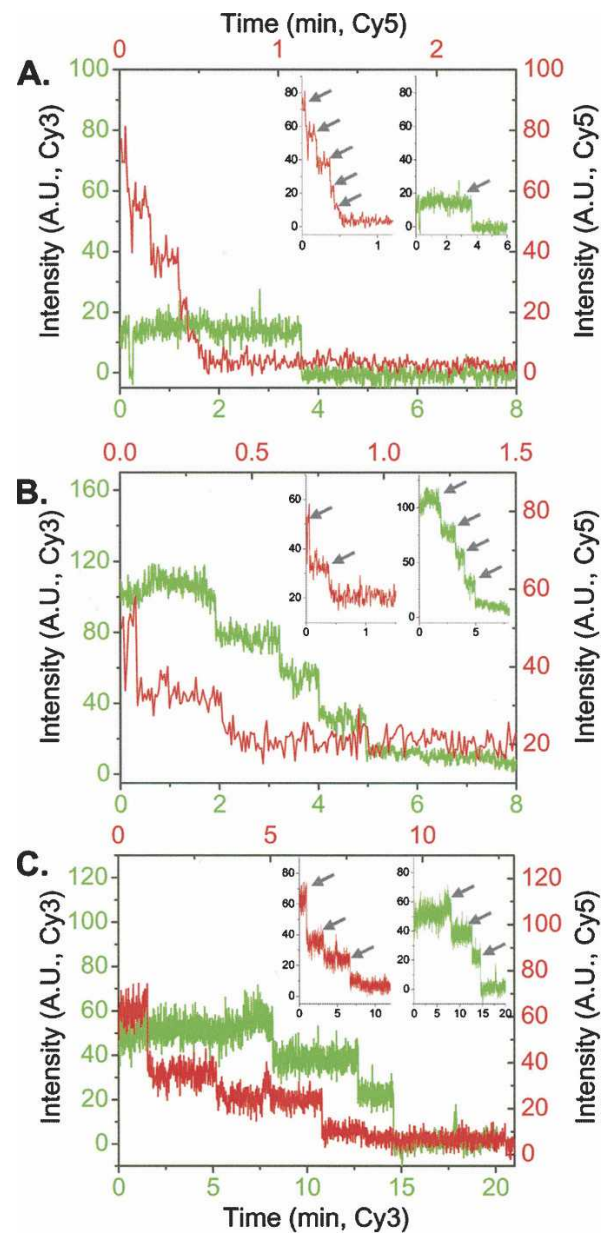

FIGURE 8. Photobleaching curves of dual-labeled procapsid/pRNA complexes assembled from both Cy3- and Cy5-labeled individual pRNA building blocks (Guo et al. 1998). (A) One Cy3-pRNA and five Cy5-pRNAs; $(B)$ two Cy3-pRNAs and four Cy5-pRNAs; $(C)$ three Cy3-pRNAs and three Cy5-pRNAs. (Insets) Clearer views of the individual colored plots. (Gray arrows) Indicate where the fluorescence intensity drops (photobleaching steps). faster than $\mathrm{Cy} 3$. Thus, the time scales of the curves for $\mathrm{Cy} 3$ and $\mathrm{Cy} 5$ are different. It is also noticeable that in Figure $8 \mathrm{~B}$, the baseline in the photobleaching curve for Cy5-pRNA does not reach zero. This is caused by the cross-talk of the signals from the Cy3 channel to the Cy5 channel, which is due to the broad fluorescence emission spectra of Cy3.

\section{Comparison of different methods for single-fluorophore labeling of RNA}

Although the main body of our fluorescence experiments on pRNA has involved the use of Cy3-AMP (F550/570; AdeGenix) and Cy5-AMP (F650/670) as transcription initiators under the $\phi 2.5$ promoter by T7 RNA polymerase (Li et al. 2005), other fluorescence labeling methods, as summarized below, have also been successfully applied.

Chemical synthesis of 5'-Cy3- or Cy5-labeled DNA fragments (IDT), followed by complementation with a region of unlabeled $\phi 29 \mathrm{pRNA}$, is an efficient and low cost method to prepare a fluorescent DNA-RNA hybrid, which may function like fluorescent pRNA.

Alternatively, functional pRNA can be assembled by two or three RNA fragments (Fang et al. 2005). Thus, fluorescent pRNA can be assembled from RNA fragments with one of them containing a single fluorophore of Cy3 or Cy5 (Dharmacon).

All three fluorophore labeling methods work well for the preparation of pRNA with a single fluorophore. All Cy3 (or its equivalent F550/570) labeled products are stable enough to allow sufficient time for the detection of individual photobleaching steps. However, photoinduced blinking and fast photobleaching of Cy5 (or its equivalent, F650/ 670) made it difficult to obtain unequivocal results from Cy3/Cy5 dual-color analysis. Using Trolox in an oxygenscavenging system can reduce the problem of photoinduced blinking, and dramatically extend the fluorescence photoncounting time before photobleaching (Rasnik et al. 2006). As a result, dual labeling of pRNA Cy3/Cy5 pairs significantly improves the signal stability and linearity in dualcolor imaging.

We have demonstrated that this single-fluorophore dualviewing system is useful in quantification of fluorescent nanoparticles. The photobleaching assay for direct counting is not limited by the optical resolution. The stoichiometry of the fluorescent nanoparticles determined by the photobleaching assay was congruent with the statistical prediction from the binomial distribution (Shu et al. 2007). The imaging system could be used in counting RNA molecules in other biological complexes, such as the ribosome. The dual-viewing system will enable excitation and recording at different wavelengths simultaneously. This capability could be utilized to discriminate different complexes with distinctive labels and could be useful for the determination of the relative motion of two components with different labels in motion machines in real time. 


\section{MATERIALS AND METHODS}

\section{Labeling pRNA with Cy3 or Cy5}

The pRNA was labeled with a single Cy3 or Cy5 to each pRNA molecule by RNA transcription in the presence of ADO F550/570 or ADO F650/670 (AdeGenix, Inc.), respectively. During transcription, the labeled AMP could only be used for initiation of transcription by the T7 RNA polymerase (He et al. 1997), but could not be used for RNA chain extension since chain extension requires the presence of a triphosphate, such as ATP (Garver and Guo 1997; Huang 2003; Huang et al. 2003; Li et al. 2005; Guo et al. 2006). The resultant 117-base pRNA has a single Cy3 or Cy5 molecule tagged to its $5^{\prime}$ end. The labeling efficiency of pRNA was obtained from its UV/Vis absorbance spectrum. RNA concentration was determined by $\mathrm{OD}_{260}$ and converted to molar concentration $\left(1 \mathrm{OD}_{260}=40 \mathrm{ng} / \mu \mathrm{L}=1 \mu \mathrm{M}\right)$. The molar concentration of Cy3 was determined from the absorbance at $550 \mathrm{~nm}\left(\varepsilon_{550}=\right.$ $\left.150,000 \mathrm{~mol}^{-1} \mathrm{~cm}^{-1}\right)$, while the molar concentration of Cy5 was determined from the absorbance at $650 \mathrm{~nm}\left(\varepsilon_{650}=250,000 \mathrm{~mol}^{-1}\right.$ $\mathrm{cm}^{-1}$ ). Labeling efficiency was calculated as [Cy3 or Cy5]/[RNA].

\section{Assembly of fluorescent nanoparticles and nanomotors}

The nanoparticles of His-gp16/pRNA complexes were assembled by mixing $0.2 \mu \mathrm{g}$ of His-gp 16 with $0.08 \mu \mathrm{g}$ of fluorescent pRNA in $10 \mu \mathrm{L}$ of PEG buffer (Huang and Guo 2003a). The mixture was incubated for $30 \mathrm{~min}$ at room temperature and then immobilized onto the surface of the perfusion chamber after 1:500 dilution in TMS (50 mM Tris at pH 8.0, $100 \mathrm{mM} \mathrm{NaCl}, 10 \mathrm{mM} \mathrm{MgCl}_{2}$ ).

The nanomotors of procapsid/pRNA complexes were prepared using fluorescent-labeled pRNA and isolated from the free pRNA in solution as previously reported (Chen et al. 2000).

\section{Fluorescence microscopy}

The surface of the perfusion chamber was coated with anti- $\phi 29$ or anti-His-gp16 IgG (Proteintech Group, Inc) by incubation of $0.1 \mathrm{mg} / \mathrm{mL}$ IgG in PBS (phosphate buffered saline at $\mathrm{pH}$ 8.0) overnight at $4^{\circ} \mathrm{C}$. The fluorescent His-gp 16/pRNA or procapsid/pRNA complexes were then immobilized to the surface by $30 \mathrm{~min}$ of incubation at room temperature. After washing with TMS, an oxygen depletion solution containing glucose oxidase, catalase, $\beta$-D-glucose, and 2-mercaptoethanol (Ha et al. 2002) was infused in before observation. An inverted Olympus microscope (IX71) with an oil immersion objective (PlanoApo, $60 \times, \mathrm{NA}=1.4$ ) was used. Signals were collected by an EMCCD camera (iXon 877V; Andor Technology). During image recording, a laser of $532 \mathrm{~nm}$ was used for Cy3 excitation and a laser of $638 \mathrm{~nm}$ was used for Cy5 excitation. The exposure time was set to be $0.4 \mathrm{sec}$, with a time interval of $0.6 \mathrm{sec}$ between frames. In dual-color fluorescence imaging, both lasers were used simultaneously, with signals recorded through the dual-view imager (Optical Insights, LLC).

\section{ACKNOWLEDGMENTS}

We thank Taekjip Ha, Wulf-Dieter Moll, and Mark Browne (Andor Technolgoy) for their technical assistance on TIR setup. We also thank David Rueda, Nils Walter, Chris Meiners, Meredith
Lambert, Peter Stockley, Eckhard Jankowsky, and Toshio Yanagida (Japan) for their valuable comments and other technical assistance. This research was supported by NIH grants R01GM59944 and R01-EB03730.

Received March 12, 2007; accepted July 10, 2007.

\section{REFERENCES}

Abels, J.A., Moreno-Herrero, F., van der Heijden, T., Dekker, C.F., and Dekker, N.H. 2005. Single-molecule measurements of the persistence length of double-stranded RNA. Biophys. J. 88: 27372744.

Adachi, K., Yasuda, R., Noji, H., Itoh, H., Harada, Y., Yoshida, M., and Kinosita Jr., K. 2000. Stepping rotation of F1-ATPase visualized through angle-resolved single- fluorophore imaging. Proc. Natl. Acad. Sci. 97: 7243-7247.

Ambrose, W.P., Goodwin, P.M., and Nolan, J.P. 1999. Singlemolecule detection with total internal reflection excitation: Comparing signal-to-background and total signals in different geometries. Cytometry 36: 224-231.

Balci, H., Ha, T., Sweeney, H.L., and Selvin, P.R. 2005. Interhead distance measurements in myosin VI via SHRImP support a simplified hand-over-hand model. Biophys. J. 89: 413-417.

Block, S.M., Goldstein, L.S., and Schnapp, B.J. 1990. Bead movement by single kinesin molecules studied with optical tweezers. Nature 348: $348-352$.

Chen, C. and Guo, P. 1997. Sequential action of six virus-encoded DNA-packaging RNAs during phage $\phi 29$ genomic DNA translocation. J. Virol. 71: 3864-3871.

Chen, C., Sheng, S., Shao, Z., and Guo, P. 2000. A dimer as a building block in assembling RNA. A hexamer that gears bacterial virus $\$ 29$ DNA-translocating machinery. J. Biol. Chem. 275: 17510-17516.

Chu, S. 2003. Biology and polymer physics at the single-molecule level. Philos. Transact. A Math. Phys. Eng. Sci. 361: 689-698.

Dohoney, K.M. and Gelles, J. 2001. $\chi$-sequence recognition and DNA translocation by single RecBCD helicase/nuclease molecules. Nature 409: 370-374.

Elias, A.L., Rodriguez-Manzo, J.A., McCartney, M.R., Golberg, D., Zamudio, A., Baltazar, S.E., Lopez-Urias, F., Munoz-Sandoval, E., Gu, L., Tang, C.C., et al. 2005. Production and characterization of single-crystal FeCo nanowires inside carbon nanotubes. Nano Lett. 5: $467-472$.

Fang, Y., Cai, Q., and Qin, P.Z. 2005. The procapsid binding domain of $\$ 29$ packaging RNA has a modular architecture and requires 2 '-hydroxyl groups in packaging RNA interaction. Biochemistry 44: 9348-9358.

Finer, J.T., Simmons, R.M., and Spudich, J.A. 1994. Single myosin molecule mechanics: Piconewton forces and nanometre steps. Nature 368: 113-119.

Fu, A., Micheel, C.M., Cha, J., Chang, H., Yang, H., and Alivisatos, A.P. 2004. Discrete nanostructures of quantum dots/Au with DNA. J. Am. Chem. Soc. 126: 10832-10833.

Funatsu, T., Harada, Y., Tokunaga, M., Saito, K., and Yanagida, T. 1995. Imaging of single fluorescent molecules and individual ATP turnovers by single myosin molecules in aqueous solution. Nature 374: $555-559$.

Garver, K. and Guo, P. 1997. Boundary of pRNA functional domains and minimum pRNA sequence requirement for specific connector binding and DNA packaging of phage $\phi 29$. RNA 3: 1068-1079.

Gordon, M.P., Ha, T., and Selvin, P.R. 2004. Single-molecule highresolution imaging with photobleaching. Proc. Natl. Acad. Sci. 101: $6462-6465$.

Gould, S., Marti, O., Drake, B., Hellemans, L., Bracker, C.E., Hansma, P.K., Keder, N.L., Eddy, M.M., and Stucky, G.D. 1988. Molecular resolution images of amino-acid crystals with the atomic force microscope. Nature 332: 332-334. 
Guo, P., Zhang, C., Chen, C., Trottier, M., and Garver, K. 1998. Inter-RNA interaction of phage $\$ 29$ pRNA to form a hexameric complex for viral DNA transportation. Mol. Cell 2: 149-155.

Guo, S., Huang, F., and Guo, P. 2006. Construction of folateconjugated pRNA of bacteriophage $\$ 29$ DNA packaging motor for delivery of chimeric siRNA to nasopharyngeal carcinoma cells. Gene Ther. 13: 814-820.

Ha, T., Zhuang, X., Kim, H.D., Orr, J.W., Williamson, J.R., and Chu, S. 1999. Ligand-induced conformational changes observed in single RNA molecules. Proc. Natl. Acad. Sci. 96: 9077-9082.

Ha, T., Rasnik, I., Cheng, W., Babcock, H.P., Gauss, G.H., Lohman, T.M., and Chu, S. 2002. Initiation and reinitiation of DNA unwinding by the Escherichia coli Rep helicase. Nature 419: 638-641.

He, B., Rong, M., Lyakhov, D., Gartenstein, H., Diaz, G., Castagna, R., McAllister, W.T., and Durbin, R.K. 1997. Rapid mutagenesis and purification of phage RNA polymerases. Protein Expr. Purif. 9: 142-151.

He, Y., Li, H.W., and Yeung, E.S. 2005. Motion of single DNA molecules at a liquid-solid interface as revealed by variable-angle evanescent-field microscopy. J. Phys. Chem. B 109: 8820-8832.

Hoeprich, S. and Guo, P. 2002. Computer modeling of threedimensional structure of DNA-packaging RNA (pRNA) monomer, dimer, and hexamer of $\phi 29$ DNA packaging motor. J. Biol. Chem. 277: 20794-20803.

Huang, F. 2003. Efficient incorporation of CoA, NAD, and FAD into RNA by in vitro transcription. Nucleic Acids Res. 31: e8. doi: 10.1093/nar/gng008.

Huang, L.P. and Guo, P. 2003a. Use of acetone to attain highly active and soluble DNA packaging protein gp16 of $\phi 29$ for ATPase assay. Virology 312: 449-457.

Huang, L.P. and Guo, P. 2003b. Use of PEG to acquire highly soluble DNA-packaging enzyme gp16 of bacterial virus $\$ 29$ for stoichiometry quantification. J. Virol. Methods 109: 235-244.

Huang, F., Wang, G., Coleman, T., and Li, N. 2003. Synthesis of adenosine derivatives as transcription initiators and preparation of $5^{\prime}$ fluorescein- and biotin-labeled RNA through one-step in vitro transcription. RNA 9: 1562-1570.

Ibarra, B., Caston, J.R., Llorca, O., Valle, M., Valpuesta, J.M., and Carrascosa, J.L. 2000. Topology of the components of the DNA packaging machinery in the phage $\phi 29$ prohead. J. Mol. Biol. 298: 807-815.

Iwane, A.H., Funatsu, T., Harada, Y., Tokunaga, M., Ohara, O., Morimoto, S., and Yanagida, T. 1997. Single molecular assay of individual ATP turnover by a myosin-GFP fusion protein expressed in vitro. FEBS Lett. 407: 235-238.

Johnson, D.S., Bai, L., Smith, B.Y., Patel, S.S., and Wang, M.D. 2007. Single-molecule studies reveal dynamics of DNA unwinding by the ring-shaped T7 helicase. Cell 129: 1299-1309.

Joo, C., McKinney, S.A., Nakamura, M., Rasnik, I., Myong, S., and Ha, T. 2006. Real-time observation of RecA filament dynamics with single monomer resolution. Cell 126: 515-527.

Kastner, B., Bach, M., and Luhrmann, R. 1991. Electron microscopy of U4/U6 snRNP reveals a Y-shaped U4 and U6 RNA containing domain protruding from the U4 core RNP. J. Cell Biol. 112: 10651072.

Kibsgaard, J., Lauritsen, J.V., Laegsgaard, E., Clausen, B.S., Topsoe, H., and Besenbacher, F. 2006. Cluster-support interactions and morphology of MoS2 nanoclusters in a graphitesupported hydrotreating model catalyst. J. Am. Chem. Soc. 128: 13950-13958.

Lee, T.J. and Guo, P. 2006. Interaction of gp16 with pRNA and DNA for genome packaging by the motor of bacterial virus $\phi 29$. J. Mol. Biol. 356: 589-599.

Li, N., Yu, C., and Huang, F. 2005. Novel cyanine-AMP conjugates for efficient 5' RNA fluorescent labeling by one-step transcription and replacement of $\left[\gamma^{32} \mathrm{P}\right]$ ATP in RNA structural investigation. Nucleic Acids Res. 33: e37. doi: 10.1093/nar/gni036.
Lu, H.P., Xun, L., and Xie, X.S. 1998. Single-molecule enzymatic dynamics. Science 282: 1877-1882.

Marti, O., Ribi, H.O., Drake, B., Albrecht, T.R., Quate, C.F., and Hansma, P.K. 1988. Atomic force microscopy of an organic monolayer. Science 239: 50-52.

Mikkelsen, A., Skold, N., Ouattara, L., Borgstrom, M., Andersen, J.N., Samuelson, L., Seifert, W., and Lundgren, E. 2004. Direct imaging of the atomic structure inside a nanowire by scanning tunnelling microscopy. Nat. Mater. 3: 519-523.

Pante, N., Jarmolowski, A., and Izaurralde, E.E.A. 1997. Visualizing nuclear export of different classes of RNA by electron microscopy. RNA 3: 498-513.

Qu, X.H., Wu, D., Mets, L., and Scherer, N.F. 2004. Nanometerlocalized multiple single-molecule fluorescence microscopy. Proc. Natl. Acad. Sci. 101: 11298-11303.

Rasnik, I., McKinney, S.A., and Ha, T. 2006. Nonblinking and longlasting single-molecule fluorescence imaging. Nat. Methods 3: 891893.

Rauer, B., Neumann, E., Widengren, J., and Rigler, R. 1996. Fluorescence correlation spectrometry of the interaction kinetics of tetramethylrhodamin $\alpha$-bungarotoxin with Torpedo californica acetylcholine receptor. Biophys. Chem. 58: 3-12.

Rueda, D., Bokinsky, G., Rhodes, M.M., Rust, M.J., Zhuang, X., and Walter, N.G. 2004. Single-molecule enzymology of RNA: Essential functional groups impact catalysis from a distance. Proc. Natl. Acad. Sci. 101: 10066-10071.

Shu, D., Zhang, H., Jin, J., and Guo, P. 2007. Counting of six pRNAs of $\$ 29$ DNA-packaging motor with customized single molecule dual-view system. EMBO J. 26: 527-537.

Sosa, H., Peterman, E.J., Moerner, W.E., and Goldstein, L.S. 2001. ADP-induced rocking of the kinesin motor domain revealed by single-molecule fluorescence polarization microscopy. Nat. Struct. Biol. 8: $540-544$

Svoboda, K. and Block, S.M. 1994. Force and velocity measured for single kinesin molecules. Cell 77: 773-784.

Tokunaga, M., Kitamura, K., Saito, K., Iwane, A.H., and Yanagida, T. 1997. Single molecule imaging of fluorophores and enzymatic reactions achieved by objective-type total internal reflection fluorescence microscopy. Biochem. Biophys. Res. Commun. 235: 47-53.

Vale, R.D., Funatsu, T., Pierce, D.W., Romberg, L., Harada, Y., and Yanagida, T. 1996. Direct observation of single kinesin molecules moving along microtubules. Nature 380: 451-453.

Weiss, S. 1999. Fluorescence spectroscopy of single biomolecules. Science 283: 1676-1683.

Xie, Z., Srividya, N., Sosnick, T.R., Pan, T., and Scherer, N.F. 2004. Single-molecule studies highlight conformational heterogeneity in the early folding steps of a large ribozyme. Proc. Natl. Acad. Sci. 101: $534-539$.

Xu, X.H. and Yeung, E.S. 1997. Direct measurement of singlemolecule diffusion and photodecomposition in free solution. Science 275: 1106-1109.

Yan, H., Saiani, A., Gough, J.E., and Miller, A.F. 2006. Thermoreversible protein hydrogel as cell scaffold. Biomacromolecules 7: 27762782.

Yildiz, A., Forkey, J.N., McKinney, S.A., Ha, T., Goldman, Y.E., and Selvin, P.R. 2003. Myosin V walks hand-over-hand: single fluorophore imaging with 1.5-nm localization. Science 300: 2061-2065.

Zhang, F., Lemieux, S., Wu, X., St.-Arnaud, S., McMurray, C.T., Major, F., and Anderson, D. 1998. Function of hexameric RNA in packaging of bacteriophage $\$ 29$ DNA in vitro. Mol. Cell 2: 141147.

Zhuang, X., Bartley, L.E., Babcock, H.P., Russell, R., Ha, T., Herschlag, D., and Chu, S. 2000. A single-molecule study of RNA catalysis and folding. Science 288: 2048-2051.

Zhuang, X., Kim, H., Pereira, M.J., Babcock, H.P., Walter, N.G., and Chu, S. 2002. Correlating structural dynamics and function in single ribozyme molecules. Science 296: 1473-1476. 

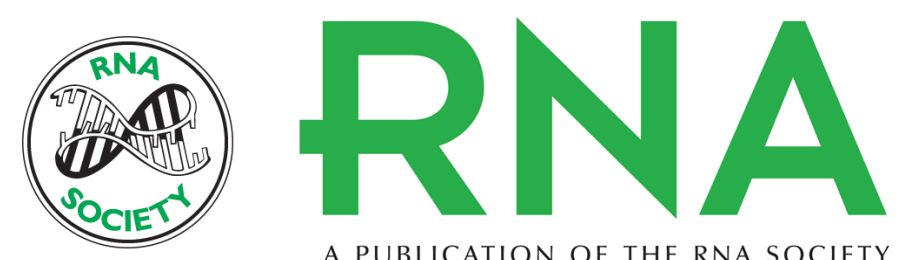

A PUBLICATION OF THE RNA SOCIETY

\section{Instrumentation and metrology for single RNA counting in biological complexes or nanoparticles by a single-molecule dual-view system}

Hui Zhang, Dan Shu, Faqing Huang, et al.

RNA 2007 13: 1793-1802 originally published online August 13, 2007

Access the most recent version at doi:10.1261/rna.587607

$\begin{array}{ll}\text { References } & \begin{array}{l}\text { This article cites } 57 \text { articles, } 20 \text { of which can be accessed free at: } \\ \text { http://rnajournal.cshlp.org/content/13/10/1793.full.html\#ref-list-1 }\end{array}\end{array}$

License

Email Alerting Receive free email alerts when new articles cite this article - sign up in the box at the Service top right corner of the article or click here.

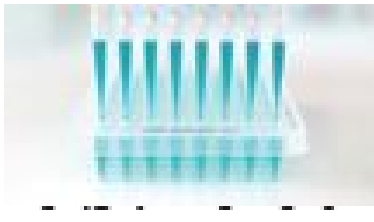

Providing Precise Solutions for your research.

To subscribe to RNA go to:

http://rnajournal.cshlp.org/subscriptions 